\title{
Light Scattering by Deformation of the Plane Texture of Smectic and Cholesteric Liquid Crystals
}

\section{Citation}

Clark, Noel A., and Peter S. Pershan. 1973. Light scattering by deformation of the plane texture of smectic and cholesteric liquid crystals. Physical Review Letters 30(1): 3-6.

\section{Published Version}

doi:10.1103/PhysRevLett.30.3

\section{Permanent link}

http://nrs.harvard.edu/urn-3:HUL.InstRepos:10361960

\section{Terms of Use}

This article was downloaded from Harvard University's DASH repository, and is made available under the terms and conditions applicable to Other Posted Material, as set forth at http:// nrs.harvard.edu/urn-3:HUL.InstRepos:dash.current.terms-of-use\#LAA

\section{Share Your Story}

The Harvard community has made this article openly available.

Please share how this access benefits you. Submit a story.

Accessibility 


\title{
PHYSICAL REVIEW LETTERS
}

\begin{tabular}{lcc}
\hline \hline Volume 30 & 1 JANUARY 1973 & Number 1 \\
\hline \hline
\end{tabular}

\section{Light Scattering by Deformation of the Plane Texture of Smectic and Cholesteric Liquid Crystals*}

\author{
Noel A. Clark and P. S. Pershan \\ Division of Engineering and Applied Physics, Harvard University, Cambridge, Massachusetts 02138 \\ (Received 18 October 1972)
}

\begin{abstract}
Distortions of the plane texture of smectic- $A$ and cholesteric liquid crystals are shown to combine with the uniaxial birefringence of the plane texture to produce a strong characteristic light-scattering effect. We have observed static deformations, arising from surface defects, as well as dynamic deformations arising from thermal or mechanical stress. An immediate application of this effect is to confirm theoretical speculations on the elasticity of smectic- $A$ phases.
\end{abstract}

Theoretical work, firstly by de Gennes ${ }^{1}$ and subsequently by others, ${ }^{2}$ suggests that for an incompressible smectic- $A$ liquid crystal in the linear hydrodynamic approximation, the elastic strain can be described in terms of a single variable $u(x, y, z)$ that specifies local displacements of the smectic layers similar to the manner in which the strains in single crystals are described in terms of the displacement of lattice points. The theoretical prediction is that away from boundaries and localized defects and in the absence of externally applied body forces, $u(x, y, z)$ satisfies the differential equation

$$
\left\{b \frac{\partial^{2}}{\partial z^{2}}-K\left[\frac{\partial^{2}}{\partial x^{2}}+\frac{\partial^{2}}{\partial y^{2}}\right]^{2}\right\} u(x, y, z)=0,
$$

where the $z$ axis is taken normal to the smectic layers. For the incompressible isothermal smectic $^{2} b$ is the single first-order elastic constant required and $K$ is the single Frank-like secondorder elastic constant. ${ }^{1}$ Although Eq. (1) is qualitatively consistent with observations on the gross morphology of smectic- $A$ systems, there have not been any serious attempts to test it more quantitatively. We present here first observations on a new optical effect which will allow quantitative study and which has enabled us to place an upper limit on the ratio $K / b$ for a smectic- $A$ sample. Experimental data are reported on a racemic mixture of $p$-butoxybenzal- $p$ - $(\beta$-methylbutyl) aniline $(\mathrm{BBMBA})^{3}$ at $20^{\circ} \mathrm{C}$. This compound is nematic from 58 to $37^{\circ} \mathrm{C}$, smectic $A$ from 37 to $18^{\circ} \mathrm{C}$, and has smectic $-B$ and crystalline phases at lower temperatures.

Single-domain smectic- $A$ samples in the planar texture were produced between two glass slides separated by amounts varying from 25 to $750 \mu \mathrm{m}$ by cooling from the nematic. The samples were observed to be optically uniaxial with a positive birefringence and optic axis normal to the glass slides. The samples showed almost no turbidity.

The experimental geometry is schematically shown in Fig. 1(a). A collimated beam $L S$ from a $20-\mathrm{mW} \mathrm{He}-\mathrm{Ne}$ laser $(6328 \AA)$ is incident on a smectic- $A$ sample at an angle $\varphi_{i}$ to the uniaxial (or $z$ ) direction in the $y-z$ plane. The illustration in Fig. 1(a) assumes that the light is polarized perpendicular to the principal plane (in the $x$ direction) such that it propagates as an ordinary wave in the sample. For $\varphi_{i}=0$, only the transmitted laser beam, striking the observation plane at point $O$, is visible. However, for $\varphi_{i} \neq 0$ we observe, in addition to this spot, a bright crescent of scattered light, $A B C$, polarized locally nor- 


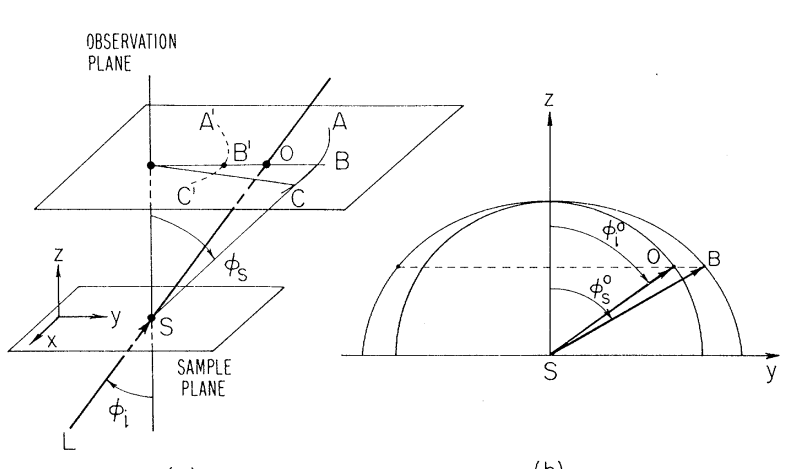

(a)

FIG. 1. (a) Schematic of the experimental geometry. The angles $\varphi_{i}$ and $\varphi_{s}$ are measured outside the sample. (b) Incident and scattered wave vectors measured inside the sample subject to the condition $k_{z}^{i}=k_{z} s$.

mal to the crescent. The crescent consists of a speckled pattern of coherence areas, which are fixed in time, indicating that the scattering is produced by microscopic inhomogeneities which are also fixed in time. Microscopic observations of the scattered light, obtained with a polarizing microscope using tilted samples and oblique illumination, showed that the detailed spatial distribution of scattered light remained unchanged under conditions where the sample was made to flow between the slides. This suggests that, in an equilibrium sample, the scattering originates from defects which are pinned to the surface. The geometry and polarization of the crescent is completely described by assuming static dielectric constant inhomogeneities $\delta \vec{\epsilon}(\boldsymbol{r})$ that depend on only $x$ and $y$ so that their Fourier transforms $\delta \overrightarrow{\boldsymbol{\epsilon}}\left(q_{x}, q_{y}, q_{z}\right)$ are sharply peaked at $q_{z}=0$. Thus, an incident light beam of wave vector $\overrightarrow{\mathrm{k}}^{i}$ $=\left(k_{x}{ }^{i}, k_{y}{ }^{i}, k_{z}{ }^{i}\right)$ is elastically scattered into a wave of wave vector $\overrightarrow{\mathrm{k}}^{s}=\left(k_{x}{ }^{i}+q_{x}, k_{y}{ }^{i}+q_{y}, k_{z}{ }^{i}\right)$. Figure 1 (b) illustrates the wave vectors of the scattered light (inside the sample) allowed under the condition that $q_{z}=0$. The line $S O$ is the wave vector (inside the sample) for the incident light, polarized as an ordinary wave. The wave vector for an extraordinary wave of the same frequency and $z$ component of wave vector, $S B$, is confined to a cone of apex angle $\varphi_{s}^{0}$ (as measured inside the sample) leading to circular crescents in accord with our observations. Figure 2 demonstrates the quantitative agreement between the cone angles $\varphi_{s}$ measured outside the sample, and those calculated assuming $q_{z}=0$ and using the measured values of the refractive indices of $\mathrm{BBMBA}^{3}\left(n_{e}\right.$ $=1.746, n_{0}=1.507$ ). Results are presented for

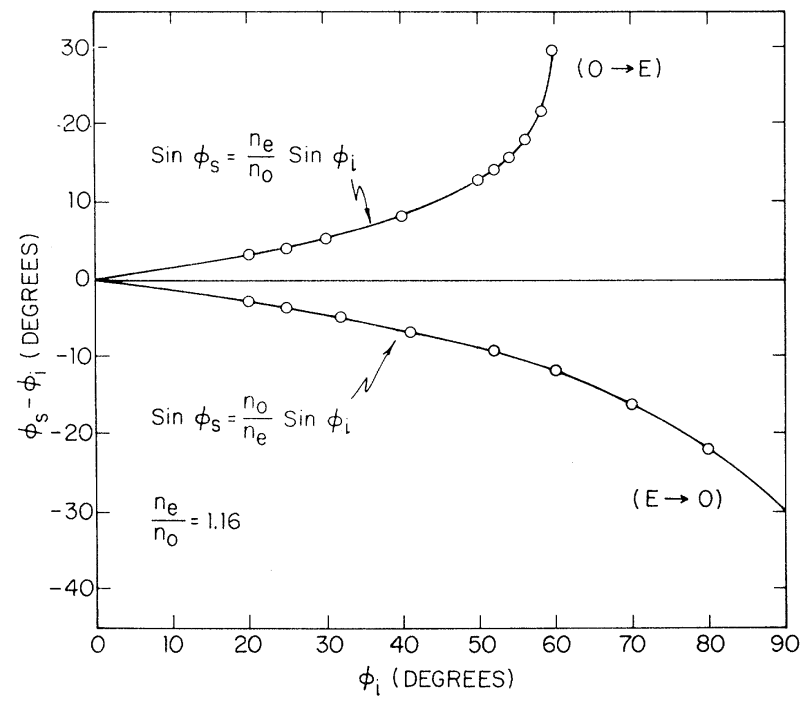

FIG. 2. Cone angles $\varphi_{s}$ measured outside the sample of BBMBA at $20^{\circ} \mathrm{C}$ compared with values of $\varphi_{s}$ calculated using the measured refractive index ratio of BBMBA $\left(n_{e} / n_{o}=1.16\right)$.

both the geometry shown in Fig. $1(O \rightarrow E)$ and the other possibility where the incident light is an extraordinary wave and the scattered light is ordinary $(E \rightarrow O)$. In the latter case the crescent

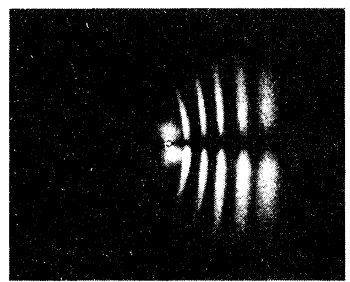

a) SMECTIC $(O \rightarrow E)$

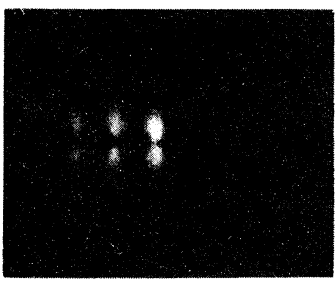

c) CHOLESTERIC $(O \rightarrow E)$

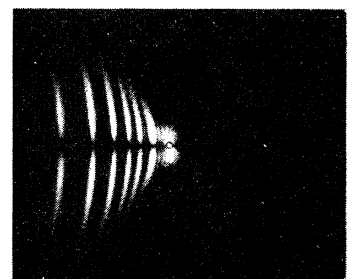

b) SMECTIC $(E \rightarrow O)$

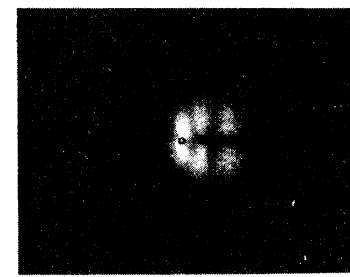

d) CHOLESTERIC $(E \rightarrow O)$
FIG. 3. Multiple-exposure photographs of the scattered-light crescents for the following angles $\varphi_{i}$ : (a) $5^{\circ}$, $25^{\circ}, 40^{\circ}, 49^{\circ}, 55^{\circ}, 58^{\circ}$; (b) $5^{\circ}, 25^{\circ}, 40^{\circ}, 50^{\circ}, 60^{\circ}, 70^{\circ}$, $80^{\circ}$; (c) $20^{\circ}, 70^{\circ}, 85^{\circ}$; (d) $10^{\circ}, 45^{\circ}, 63^{\circ}$. The circular spot at the center of each picture is the attenuated laser beam. Note the reversal of crescent position for the cholesteric since it is negatively rather than positively birefringent. 
$\left(A^{\prime} B^{\prime} C^{\prime}\right)$ of scattered light appears to the left of point $O$ in Fig. 1(a) with polarization locally tangent to the crescent. Figures $3(\mathrm{a})$ and $3(\mathrm{~b})$ show multiple-exposure photographs of scattered-light crescents obtained from a $125-\mu \mathrm{m}$-thick sample of BBMBA for a series of sample orientations $\varphi_{i}$ and the two polarization conditions.

The effects presented here can be explained in terms of static distortions in the uniform layered structure of the idealized smectic system that result from either localized defects (i.e., dislocations or disinclinations) or irregularities in the surfaces of the glass slides containing the sample. At any point $(x, y, z)$ not near to either the core of a defect or some irregular surface-boundary condition, the layers can be described in terms of the variable $u(x, y, z)$ which will locally satisfy Eq. (1). In those regions of the sample for which the local linear theory is applicable the dominant light scattering mechanism can be attributed to inhomogeneities in the optical frequency dielectric tensor, $\delta \epsilon_{x z} \sim \partial_{x} u$ and $\delta \epsilon_{y z} \sim \partial_{y} u$. For the geometry of Fig. 1(a) the intensity is roughly given by $I \sim\left\langle\left|\delta \epsilon_{x z}(\overrightarrow{\mathrm{q}})\right|^{2}\right\rangle \sim q_{x}{ }^{2}\left\langle|u(\overrightarrow{\mathrm{q}})|^{2}\right\rangle$ where $\overrightarrow{\mathrm{q}}=\left(q_{x}, q_{y}, q_{z}\right)=\overrightarrow{\mathrm{k}}^{s}-\overrightarrow{\mathrm{k}}^{i}$. The factor $q_{x}{ }^{2}$ explains the dark spots observed at point $B$ in Fig. 1 (a) and on the crescent along the $y$ axis in Fig. 3.

The selection rule $\left(\overrightarrow{\mathrm{k}}^{s}-\overrightarrow{\mathrm{k}}^{i}\right)_{z}=q_{z} \approx 0$ can be understood in terms of the following solution to Eq. (1):

$$
u(x, y, z)=\int d q_{x} d q_{y}\left\{A\left(\overrightarrow{\mathrm{q}}_{\perp}\right) \exp \left[-(K / b)^{1 / 2} q_{\perp}{ }^{2} z\right]+B\left(\overrightarrow{\mathrm{q}}_{\perp}\right) \exp \left[+(K / b)^{1 / 2} q_{\perp}^{2} z\right]\right\} \exp \left[i\left(\overrightarrow{\mathrm{q}}_{\perp} \cdot \overrightarrow{\mathrm{r}}_{\perp}\right)\right]
$$

where $\overrightarrow{\mathrm{q}}_{\perp}=\left(q_{x}, q_{y}\right)$ and $\overrightarrow{\mathrm{r}}_{\perp}=\left(r_{x}, r_{y}\right)$. The Fourier amplitudes $A\left(q_{\perp}\right)$ and $B\left(q_{\perp}\right)$ are determined by the boundary conditions on the regions for which Eq. (1) is applicable.

We have also considered and rejected alternative interpretations for the scattering mechanism. For example, suppose the scattering were due to the core regions of dislocations (which cannot be described by the linear theory). Then the resultant inhomogeneities $\delta \epsilon$ would have to be slowly varying along the $z$ axis $\left[\left(\overrightarrow{\mathrm{k}}^{s}-\overrightarrow{\mathrm{k}}^{i}\right)_{z} \approx 0\right]$, and would appear as bright lines when viewed under a microscope. Defects of this type can be observed if the samples are intentionally disturbed but are not present during our measurements.

Crude estimates of the factor $K / b$, which must have the dimension of a length squared, are $K / b$ $\sim(a / 2 \pi)^{2}$, where $a$ is the smectic layer spacing of the order of 20-30 $\AA^{1.4}$ Taking $q_{\perp} \lesssim 10^{-1}(2 \pi /$ $\lambda)$, where $\lambda=6328 \AA$, yields $(K / b)^{1 / 2} q_{\perp}^{2} \sim 5 \mathrm{~cm}^{-1}$ to be compared with $q_{\perp} \sim 10^{4} \mathrm{~cm}^{-1}$. Thus, $\delta \epsilon_{x z}(q)$ induces scattering with an uncertainty $\Delta\left(\overrightarrow{\mathrm{k}}^{i}-\overrightarrow{\mathrm{k}}^{s}\right)_{z}$ $\approx(K / b)^{1 / 2} q_{\perp}{ }^{2} \approx 0$. The length of $q_{\perp}$ is fixed by the index-of-refraction condition sketched in Fig. 1(b). Alternatively, we can measure the angular dependence of the scattered intensity normal to the crescent and determine the ratio of $K / b$. In all our experiments to date, with samples up to $750 \mu \mathrm{m}$ thick, the angular dependence has been determined by the finite sample thickness, i.e., $\Delta\left(\overrightarrow{\mathrm{k}}^{i}-\overrightarrow{\mathrm{k}}^{s}\right)_{z} \sim 2 \pi l^{-1}$, where $l$ is the sample thickness. For the $E \rightarrow 0$ polarizations this condition gives, near the point $B^{\prime}$,

$$
\begin{aligned}
& (K / b)^{1 / 2} \lesssim \lambda^{2}(2 \pi l)^{-1}\left[n_{0} /\left(n_{e}-n_{0}\right)\right]^{2}\left(\sin \varphi_{s}\right)^{-2} . \\
& \text { For } \varphi_{s} \approx 80^{\circ} \text { and } l=750 \mu \mathrm{m},(K / b)^{1 / 2} \lesssim 56 \AA .^{5}
\end{aligned}
$$

This upper limit represents the first quantitative confirmation of the structure of elastic theories proposed for smectic liquid crystals. ${ }^{1,204}$ Measurements of the relative intensity of scattered light along the crescent $(A B C)$ can be used to determine $\left\langle u\left(q_{\perp}\right)^{2}\right\rangle^{1 / 2}{ }^{6}$

Similar effects have also been observed in the cholesteric phase of $p$-ethoxybenzal- $p$ - $(\beta$-methylbutyl) aniline ${ }^{3}$ (EMBMA) which has a pitch $P$ $=2230 \AA$ at $20^{\circ} \mathrm{C}$. Photographs of the scattered light from EBMBA are shown in Figs. 3(b) and 3 (c). This is particularly interesting since theoretical arguments have been made to the effect that for $q^{-1} \gg P$, the elastic-hydrodynamic properties of cholesterics are identical with those of smectic $A,{ }^{2,7}$ the sole difference being that $(K /$ $b)^{1 / 2}$ is larger in the cholesteric. ${ }^{8}$ One consequence of this difference is the observed fact that the crescent is relatively wider in the cholesteric than in the smectic and the width can be used as some measure of $(K / b)^{1 / 2}$. Since $(b / K)^{1 / 2} q_{\perp}^{-2}$ $\gg P$, comparison can be made to the long wavelength limit of the theory for cholesterics, ${ }^{4_{0} 6}$ $(K / b)^{1 / 2}=\left(\frac{3}{8}\right)^{1 / 2} P / 2 \pi \sim 200 \AA$, where we have taken the three Frank constants equal. This is to be compared with a value of $(K / b)^{1 / 2} \approx 310 \pm 100 \AA$ deduced from the observed width of the crescents (i.e., $\delta \varphi_{s} \sim 0.5^{\circ}$ ) for $\varphi_{s}=60^{\circ}$.

We have, in addition, found the scattered light useful in detecting time-dependent distortions associated with mechanical or thermal stressing of samples confined by glass slides. We observe that an applied stress directed towards compression of the smectic layers, i.e., squeezing the slides together, reduces the intensity of light 
scattered into the crescents. Presumably this tends to straighten out the layers. McMillan ${ }^{9}$ observed an increase in layer spacing in a smectic $A$ with decrease in temperature. For fixed slide spacing, a decrease in temperature would thus produce a compressive stress, and we do observe a decrease of scattered intensity on cooling our samples. On the other hand, stresses of opposite sign, such as allowing the slides to move apart, are always accompanied by a dramatic increase in the scattered intensity and, therefore, distortion of the layers. Both reversible and irreversible distortions upon expansion have been observed. For sufficiently weak and slow expansion, the excess scattered intensity disappears when the expansion is stopped. However, continued alternate squeezing and expansion of the sample always results in a gradually increasing level of static scattering, which, microscopic study shows, is associated with enhanced static surface defects. These observations suggest the possibility of acoustic detection by expansion-induced deformations and surface defects in the smectic and cholesteric plane texture.

We would like to acknowledge the continued interest and assistance of R. Meyer and of Z. Mul- jiani and D. Dolphin who prepared the compounds studied here. We have profited from discussions with G. Durand concerning this effect. ${ }^{10}$

*Work supported in part by the National Scinece Foundation Grant No. GH-33576 and by the Division of Engineering and Applied Physics, Harvard University, Cambridge, Mass. 02138.

${ }^{1}$ P. G. de Gennes, J. Phys. (Paris), Colloq. 30, C465 (1969).

${ }^{2}$ P. C. Martin, O. Parodi, and P. S. Pershan, Phys. Rev. A $\underline{6}, 24011972$.

${ }^{3}$ D. Dolphin, Z. Muljiani, J. Cheng, and R. B. Meyer, to be published.

${ }^{4}$ F. C. Frank, Discuss. Faraday Soc. $\underline{25}, 19$ (1958).

${ }^{5}$ Alternatively, we could fit the data by a more conventional elasticity equation, $b \partial^{2} u / z^{2}+c_{44} \partial^{2} u / \partial x^{2}=0$ and obtain the result $c_{44} / b \lesssim 10^{-5}$.

${ }^{6} \mathrm{~N}$. A. Clark, to be published.

${ }^{7}$ T. C. Lubensky, Phys. Rev. A 6,452 (1972).

${ }^{8}$ For angles $\varphi_{i} \gtrsim 5^{\circ}$ the EBMBA sample behaves as an optically uniaxial system with negative birefringence $\left(n_{e} / n_{o}=0.926\right)$ (Ref. 3).

${ }^{9}$ W. L. Mc Millan, Phys. Rev. A $\underline{6}, 936$ (1972).

${ }^{10}$ Similar results to those presented here have been obtained independently and concurrently by R. Ribotta, G. Durand, and J. D. Litster (to be published) with reference to theoretical models by G. Durand (to be published) and P. G. de Gennes (to be published).

\title{
Magnetic-Field-Induced One-Dimensional Behavior in the Specific-Heat Transition in Dirty Bulk Superconductors
}

\author{
R. F. Hassing* and R. R. Hake $\dagger$ \\ Indiana University, Bloomington, Indiana 47401 \\ and \\ L. J. Barnes \\ Marlborough School, Los Angeles, California 90004
}

(Received 3 November 1972)

\begin{abstract}
The specific heat is calculated in the transition region for an extreme type-II bulk superconductor [ zero-temperature Ginzburg-Landau (GL) coherence length $\xi(0) \approx 48 \AA$, dirtiness parameter $\xi_{0} / l \approx 120$, GL parameter $\kappa \approx 67$ ] for various values of applied magnetic field $H$, and compared with previously published data. Nonlocality is neglected and the quartic term in the GL free-energy functional is treated in a Hartree approximation. The calculated and measured transitions broaden with increasing $H$, the latter in a manner suggestive of an approach to the theoretically predicted one-dimensional form.
\end{abstract}

There has recently been both theoretical ${ }^{1}$ and experimental ${ }^{2}$ interest in the question of a possible magnetic-field-induced reduction of effective dimensionality in superconductors. As noted by Lee and Shenoy, ${ }^{3}$ application of a magnetic field to a bulk superconductor can lead to effective one-dimensional behavior in the fluctuation specific heat near the critical temperature $T_{c 2}(H)$. In the presence of the field the order parameter is expanded in Landau orbitals (instead of plane 


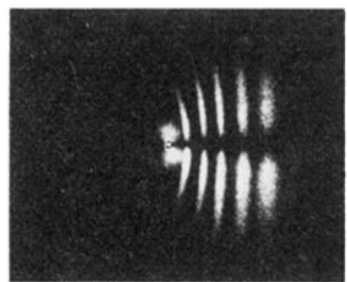

o) $\operatorname{SMECTIC~}(O \rightarrow E)$

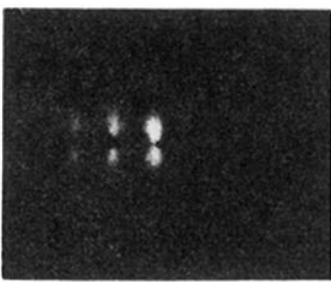

c) CHOLESTERIC $(O \rightarrow E)$

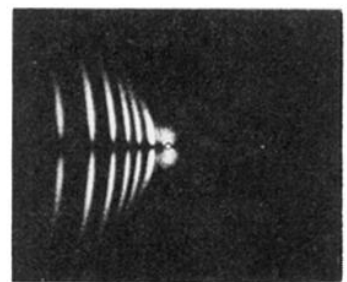

b) SMECTIC $(E \rightarrow O)$

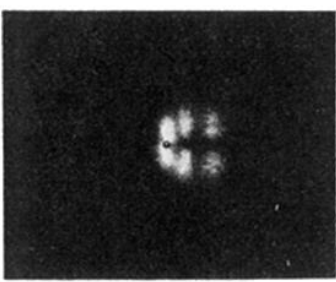

d) CHOLESTERIC $(E \rightarrow O)$

FIG. 3. Multiple-exposure photographs of the scattered-light crescents for the following angles $\varphi_{i}$ : (a) $5^{\circ}$, $25^{\circ}, 40^{\circ}, 49^{\circ}, 55^{\circ}, 58^{\circ}$; (b) $5^{\circ}, 25^{\circ}, 40^{\circ}, 50^{\circ}, 60^{\circ}, 70^{\circ}$, $80^{\circ}$; (c) $20^{\circ}, 70^{\circ}, 85^{\circ}$; (d) $10^{\circ}, 45^{\circ}, 63^{\circ}$. The circular spot at the center of each picture is the attenuated laser beam. Note the reversal of crescent position for the cholesteric since it is negatively rather than positively birefringent. 